\title{
A General One-Pot Strategy for the Synthesis of High-Performance Transparent-Conducting-Oxide Nanocrystal Inks for All-Solution- Processed Devices
}

Authors: Jizhong Song ${ }^{1,2}$, Sergei A. Kulinich ${ }^{3,4}$, Jianhai Li ${ }^{1}$, Yanli Liu ${ }^{2}$, Haibo Zeng ${ }^{1,2 *}$

Affiliations:

${ }^{1}$ Institute of Optoelectronics \& Nanomaterials, College of Materials Science and Engineering, Nanjing University of Science and Technology, Nanjing 210094, China.

${ }^{2}$ State Key Laboratory of Mechanics and Control of Mechanical Structures \& College of Materials Science and Technology, Nanjing University of Aeronautics and Astronautics, Nanjing 210016, China.

${ }^{3}$ Institute of Innovative Science and Technology, Tokai University, Kanagawa 259-1292, Japan

${ }^{4}$ School of Engineering and Applied Science, Aston University, Birmingham B4 7ET, UK

* Correspondence and requests for materials should be addressed to H.B.Z. (zeng.haibo.nano@gmail.com) 
Electrodes made of various transparent conducting oxides have been extensively used in light-emitting diodes, solar cells and thin-film transistors. However, the commercially available high-quality films are currently prepared via sputtering, which makes them not well compatible with low-cost solution-processed optoelectronics and slows down the further development of the commercial market. Here we report a generic one-pot protocol for the synthesis of diverse nanocrystals (NCs) that can be used, as inks, to prepare transparent conducting oxide films (such as ZnO, AZO, GZO, IZO, and ITO). The protocol uses inexpensive long-chain alkyl alcohols (e.g., 1-dodecanol) as reactants, and oleylamine and oleic acid as surfactants. It is relatively simple and amenable to large-scale production compared to the conventional hot-injection method. The as-synthesized NCs are shown to be easily dispersed in various organic solvents and, as such, can serve as inks for assembling thin and uniform films via solution-based processes. Largescale centimeter-wide films spin-coated by using the inks proved to be crack-free and with root-meansquare roughness values as low as $1.6 \mathrm{~nm}$. The thickness and electrical properties of the films were effectively controlled and enhanced by UV treatment. ITO films spin-coated from NCs and then UVtreated under optimal conditions and annealed at $350{ }^{\circ} \mathrm{C}$ were $300-\mathrm{nm}$ thick and showed an optimum resistivity of $112 \Omega / \mathrm{sq}$ and optical transmittance as high as $87 \%$. Such films prepared from NC inks have potential applications in various low-cost, large-area and flexible optoelectronic devices fabricated via solution processes. 


\section{Table of Contents}

Monodisperse nanocrystals of several conducting oxides (such as $\mathrm{ZnO}, \mathrm{AZO}, \mathrm{GZO}$, IZO, and ITO) were prepared via a simple, generic and easily scalable one-pot protocol. The nanocrystals are shown to be well-dispersed in various organic solvents and thus can serve as inks for assembling smooth and highly transparent films prepared via solution-based processing. The thickness and electrical properties of the nanocrystal-based films were effectively controlled and enhanced by UV treatment. After UV treatment and low- temperature annealing, the ITO nanocrystal-based films with thickness of $300 \mathrm{~nm}$ showed an optimum resistivity of $112 \Omega /$ sq and optical transmittance as high as $87 \%$. Such films have potential applications in various low-cost, large-area and flexible optoelectronic devices prepared via solution-based processes.

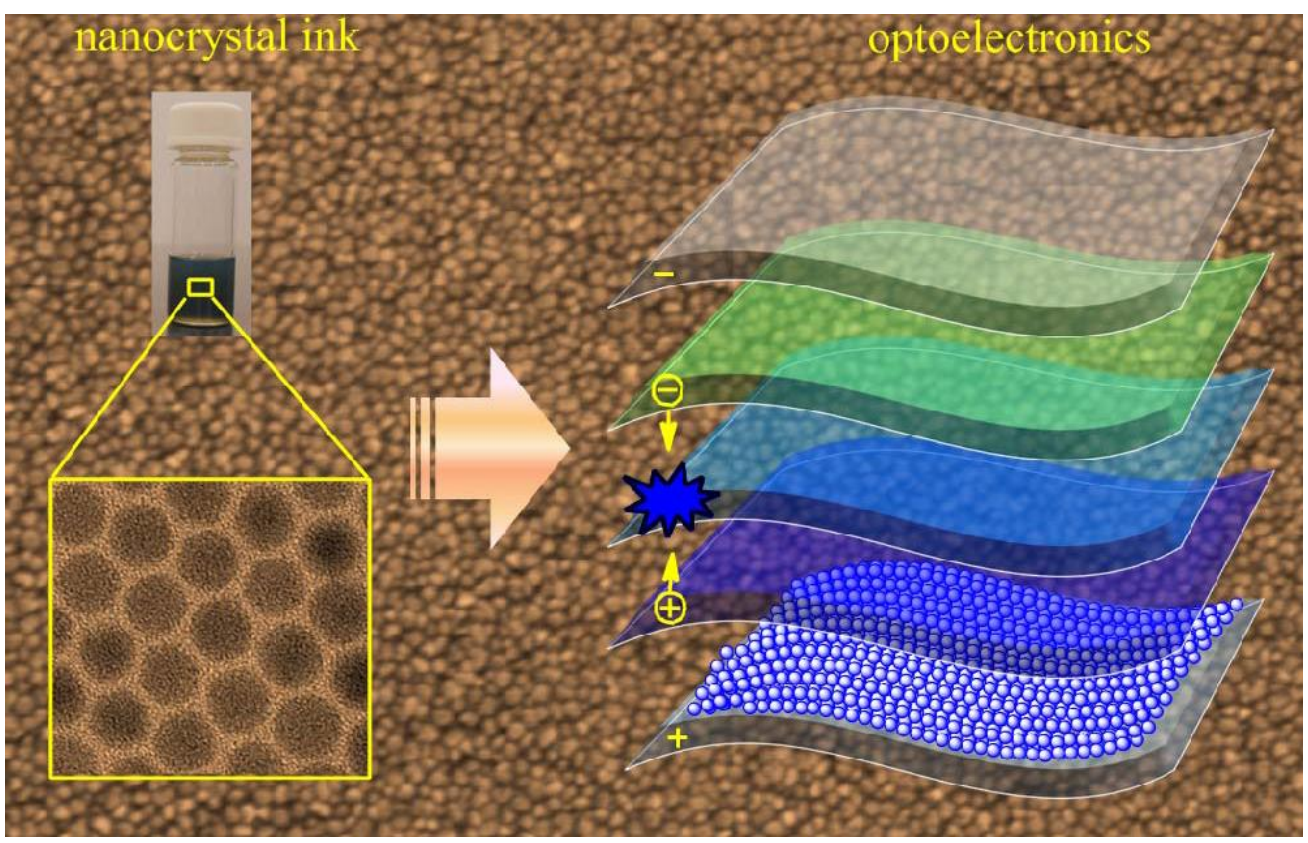




\section{Introduction}

Semiconducting optoelectronic devices (OEDs), such as light-emitting diodes (LEDs), solar cells (SCs) and thin-film transistors (TFTs), are highly anticipated in, and are already part of, modern society. Among others, solution-processed devices have received a great deal of attention as next-generation OEDs as they are often associated with low-cost and large-area fabrication ${ }^{1,2}$. Transparent electrodes, as layers of solution-processed OED structures, have been extensively investigated, the most widely used ones being tin-doped indium oxide (ITO), antimony-doped tin oxide (ATO), aluminum-doped zinc oxide (AZO) and gallium-doped zinc oxide $(\mathrm{GZO})^{3-5}$, as well as metal nanowires ( $\mathrm{Au}, \mathrm{Ag}, \mathrm{Cu}$, etc. $)^{6-8}$. Currently, the commercially available electrodes with the best electrical and optical properties are based on ITO (for LEDs or SCs) and doped $\mathrm{ZnO}$ (for SCs) ${ }^{9-12}$, which are typically deposited via relatively costly CVD or PVD processes (e.g. magnetron sputtering). While such electrodes are quite costly, the rapidly growing demand for consumer electronics, photovoltaics and smart building materials requires the development of lower-cost and high-performance transparent conducting oxides (TCOs) which are better compatible with wet-chemistry processed and flexible OEDs ${ }^{13-15}$.

Compared to sputtering, the realization of high-quality nanocrystal (NC) colloids dispersed in liquids (so-called NC inks) would be of great advantage, as the related deposition techniques, such as like spin-coating, inkjet printing and spraying, permit to prepare TCO layers on a wide variety of substrates at reasonable costs and on large areas ${ }^{16-18}$. Among other wet-chemistry approaches, preparing high-quality TCO NCs with controlled morphologies and desired properties via reactions in hot liquid media has become very popular lately ${ }^{19-21}$. Recently, highly transparent ITO films with low resistivity and GZO films with square resistivity as low as $350 \Omega /$ sq have been prepared from monodisperse ITO and GZO NCs synthesized via hot-injection, respectively ${ }^{18,22}$. The hot-injection 
method has very much gained in popularity in recent years because of its effectiveness in preparing monodisperse colloidal NCs and controlling their morphology $\mathrm{y}^{23,24}$. However, hot-injection based approaches are quite sensitive to all parameters and details during and following the mixing stage, which makes them not easily controllable and/or reproducible, especially at large scales ${ }^{20}$. Therefore, simple, one-pot and easy-to-scale-up protocols allowing for preparation of high-quality and welldispersed colloidal NCs with controlled particle size and dopant incorporation are still anticipated and a major challenge for numerous OED-related applications.

Here, we report on a facile and universal one-pot method for the synthesis of various TCO NCs that can serve as convenient inks for solution-processed OEDs. The proposed approach is amenable to scale up and uses a cheaper long-chain alkyl alcohol (1-dodecanol) when compared to those previously used by hot-injection based methods (e.g. 1,2-hexadecanediol). The as-prepared TCO NCs were welldispersed in various organic solvents (such as hexane, toluene, n-tetradecane) and efficiently used as inks to prepare smooth, crack-free, highly transparent, and highly conductive films via spin-coating. To improve the film performance, layer-by-layer assembly and UV treatment were used, after which a 300-nm-thick (2 layers) ITO film exhibited resistivity as low as $112 \Omega /$ sq and transparency as high as $87 \%$. The proposed approach is demonstrated to be easily scalable, thus being suitable for largearea film fabrication of OEDs via spinning, ink-jet printing, spraying, and roll-to-roll manufacturing techniques.

\section{Results}

Synthesis of TCO NCs. TCO NCs were synthesized via a simple one-pot approach based on heating of metal organic precursors dissolved in 1-octadecene (ODE) as a solvent, with oleylamine (OAm) and oleic acid (OA) added as surfactants, and 1-dodecanol (DDL) as reactant donating hydroxyl groups 
at a temperature of $270{ }^{\circ} \mathrm{C}$ and in nitrogen atmosphere. Compared with conventional synthesis of colloidal oxide $\mathrm{NCs},{ }^{19,} 20$ a significantly cheaper long-chain alkyl alcohol (DDL) and 1,16hexadecanediol were used for the esterification reaction. In case of $\mathrm{Zn}$ precursor, highly-crystalline hexagonal-pyramid-shaped $\mathrm{ZnO}$ NCs of about $12 \mathrm{~nm}$ in size were synthesized, as shown in Fig. 1a-c. The as-prepared NCs are uniform in size and well-dispersed due to the surfactant molecules on their surface. The HRTEM image in Fig. 1b reveals triangular and hexagonal projections (the models of hexagonal-pyramid $\mathrm{ZnO}$ are shown in Supplementary Fig. S1), corresponding to hexagonal pyramid $\mathrm{ZnO}$ NCs lying on their side or base faces. Figure 1c shows the hexagonal morphology attributed to such ZnO NCs standing on their bases. Similar hexagonal pyramid NCs with exposed crystal facets were recently reported to exhibit unique electrical properties ${ }^{25}$. As a next step, a number of $\mathrm{ZnO} \mathrm{NCs}$ doped with group III elements (AZO, GZO, and IZO) were obtained following the same procedure and simply adding different amounts of doping metal precursors (see Figs. 1d-f). The morphology of the doped and pure $\mathrm{ZnO} \mathrm{NCs}$ is seen to be very similar without any noticeable difference. Figure $1 \mathrm{~g}$ exhibits the as-synthesized IZO NCs with highly-crystalline wurtzite structure, while their STEM and elemental mapping in Fig. 1h proves that In was effectively incorporated into the $\mathrm{ZnO}$ crystal structure.

The same generic one-pot approach could also be used to prepare other TCO NCs, such as $\mathrm{Ga}_{2} \mathrm{O}_{3}$, $\mathrm{In}_{2} \mathrm{O}_{3}$, and ITO, as shown in Figs. 1i-1. The preparation of undoped $\mathrm{Ga}_{2} \mathrm{O}_{3}$ and $\operatorname{In}_{2} \mathrm{O}_{3} \mathrm{NCs}$ indirectly explains why $\mathrm{Ga}$ and In could be effectively incorporated into $\mathrm{ZnO}$ NCs. Although the reports on $\mathrm{Ga}_{2} \mathrm{O}_{3} \mathrm{NC}$ inks are scarce, recently the potential use of $\mathrm{Ga}_{2} \mathrm{O}_{3}$ in optoelectronics (e.g, in light-emitting diodes and solar-blind photodetectors) has gained interest ${ }^{26-29}$. At the same time, indium oxides, especially ITO, are widely used in optoelectronic devices, such as TFTs and transparent electrodes. The high-quality ITO NCs (displayed in Figs. 1k,1) were easily prepared through the same single-pot 
approach. Their morphology is almost the same as that of pure $\operatorname{In}_{2} \mathrm{O}_{3} \mathrm{NCs}$, while In incorporation seems to make the NC size slightly smaller. To our best knowledge, a thin film assembled from the ITO NCs reported in this work demonstrates the best conductivity ever reported in the literature and thus can be potentially used as transparent electrode for solution- processed OEDs.

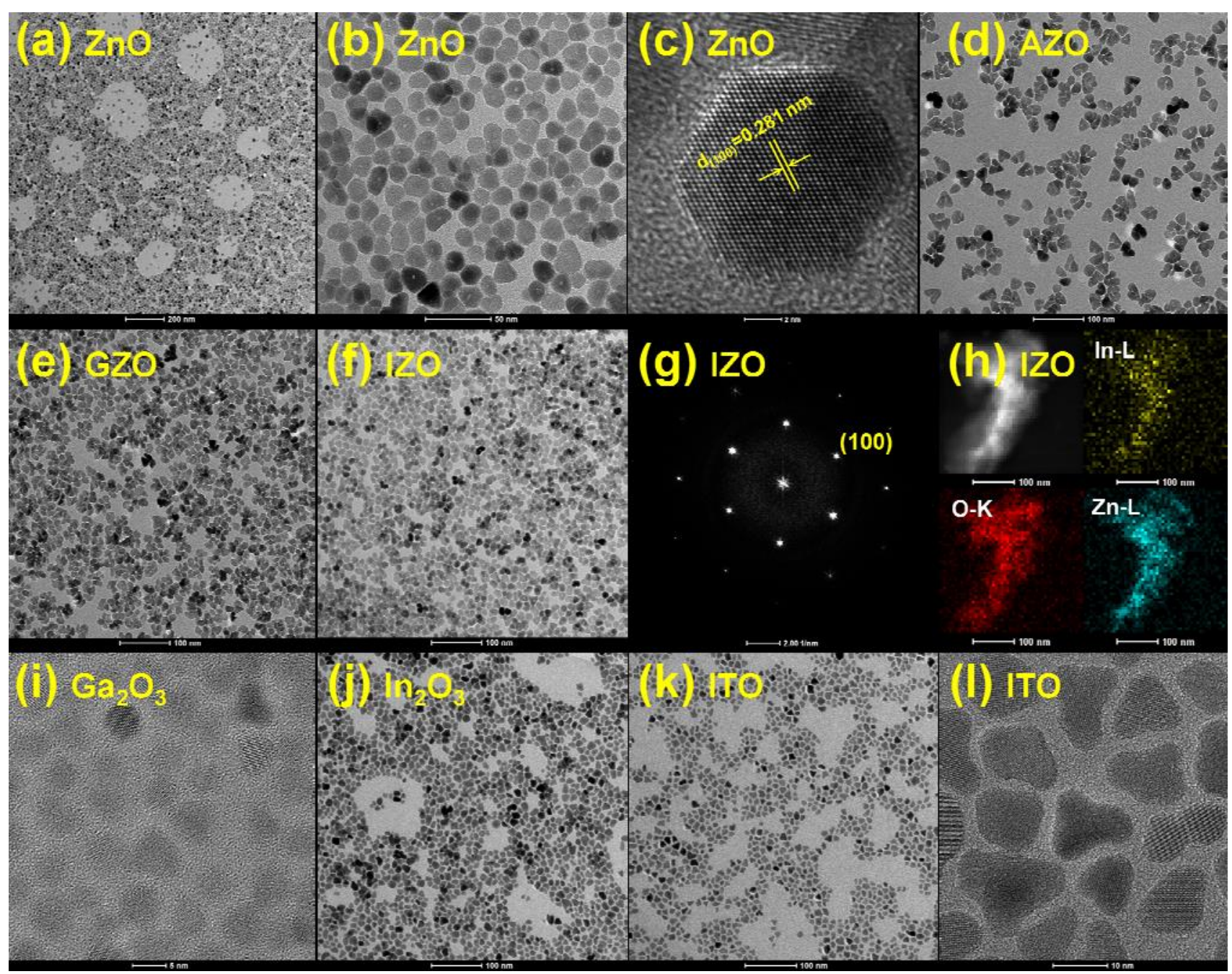

Figure 1 | TEM images of TCO NCs. (a, b) TEM images of ZnO NCs prepared in this work taken at different magnifications. (c) High-resolution TEM image of one ZnO NC. (d-f) TEM images of ZnO NCs doped with different elements: (d) AZO, (e) GZO, (f) IZO. (g) Selected area electron diffraction (SAED) pattern of IZO NCs, which is consistent with the (100) planes of the ZnO crystal structure. (h) STEM image and STEM-EDS elemental mappings of IZO NCs. (i) TEM image of $\mathrm{Ga}_{2} \mathrm{O}_{3}$ NCs. (j) TEM image of $\mathrm{In}_{2} \mathrm{O}_{3}$ NCs. (k, l) TEM images of ITO NCs taken at different magnifications. The original precursor content for AZO, GZO, IZO and ITO NCs was 1, 3, 5 and 5 at.\%, respectively. 
The reactions taking place during the NC preparation are briefly sketched in Fig. 2. The metal organic precursor (or two, in case of doped NCs) reacts with alcohol (DDL) molecules to form M-OH and ester byproducts identified by FTIR and previously reported in the literature. ${ }^{30-32}$ The conversion of metal precursors to esters in the presence of OA and OAm is believed to follow closely the kinetics previously reported by Tienes and coauthors ${ }^{33}$. The metal precursor molecules react with 1-dodecanol, producing $\mathrm{M}-\mathrm{OH}$ through ester elimination, after which metal oxides are obtained via the condensation of metal hydroxide species and release of water molecules as byproducts. Two different $\mathrm{M}-\mathrm{OH}$ species mutually react to form $\mathrm{M}-\mathrm{O}-\mathrm{M}_{1}-\mathrm{OH}$ via condensation process, which eventually gives rise to doped TCO NCs. The OA and OAm molecules act as surfactants within the entire reaction process. Since the surface of thus-obtained oxide NCs is terminated with alkyl groups, they can be easily dispersed in various organic media as inks.

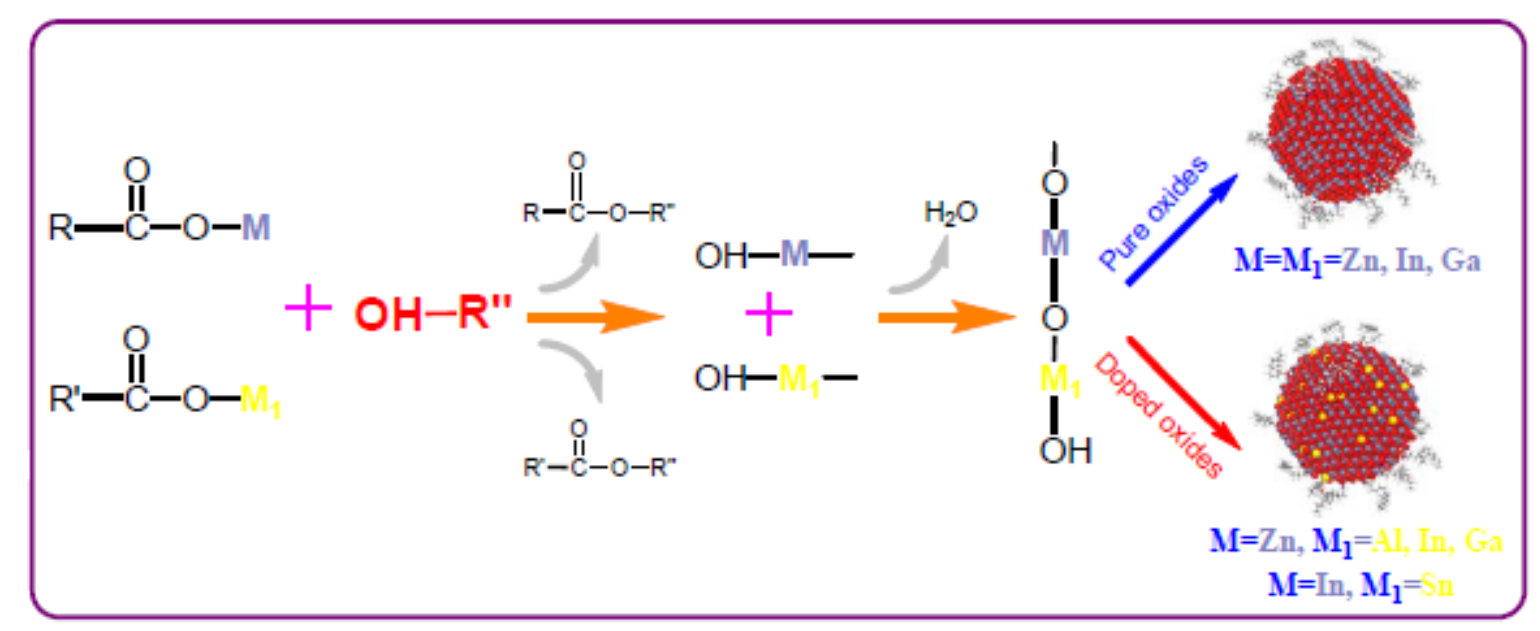

Figure 2 | Formation of TCO NCs. Schematic illustration of the processes taking place during formation of both pure and doped TCO NCs.

The surfactants (OA and OAm) and the reactant (DDL) played important roles in the process. We chose $\mathrm{ZnO}$ as a model system to demonstrate the effect of reaction parameters, such as surfactants, precursors, reaction temperature, on the product NCs. When the reaction temperature was set to 270 
${ }^{\circ} \mathrm{C}$, high-quality NCs were obtained (Fig. 1b). The NCs prepared below $270{ }^{\circ} \mathrm{C}$ had many defects and emitted visible light. For example, those prepared at $210^{\circ} \mathrm{C}$ were $\sim 5 \mathrm{~nm}$ in size (Supplementary Fig. S2) and emitted orange light characteristic of defects, while such light was barely observed from NCs prepared at $270{ }^{\circ} \mathrm{C}$ (Supplementary Fig. S3). The synthesis performed at $270{ }^{\circ} \mathrm{C}$ with OAm as a solely surfactant could also lead to NCs (Supplementary Fig. S4a), but the formed colloids were very unstable and could only be stored for at most one week. The use of OA as the only surfactant did not permit to prepare any NCs at all, even at temperatures as high as $300{ }^{\circ} \mathrm{C}$. The amount of OAm added into the system is also important and affects the product dispersion. When 1 or $2 \mathrm{~mL}$ of OAm was added, the NCs tended to aggregate (Supplementary Fig. S4b), while the addition of $3 \mathrm{~mL}$ of OAm resulted in well-dispersed ZnO NCs (Supplementary Fig. S4c,d). When $1 \mathrm{~mL}$ of DDL was used, high-quality and well-dispersed $\mathrm{ZnO}$ NCs with sizes of $\sim 12 \mathrm{~nm}$ were formed. Importantly, this reaction system turned out to be suitable for the preparation of both $\mathrm{ZnO}$ and other (undoped and doped) TCO NCs. The effect of reaction time was also tested, showing that rod-like $\mathrm{ZnO}$ formed when the system was kept under optimal conditions for $2 \mathrm{~h}$ (Supplementary Fig. S5). For the doped $\mathrm{ZnO} \mathrm{NCs}$, we took $\mathrm{Zn}(\mathrm{St})_{2}$ as a $\mathrm{Zn}$ precursor and acetylacetonates of group III metals as dopants. This choice was dictated by a relatively higher reactivity of acetylacetonates as doping precursors, which was believed to provide more effective and uniform doping ${ }^{34-37}$. When a more reactive $\mathrm{Zn}$ precursor, for instance zinc acetylacetonate, was used instead of $\mathrm{Zn}(\mathrm{St})_{2}$, as a single metal precursor or along with indium acetylacetonate, ZnO or IZO NCs could also be prepared (Supplementary Figs. S6a,b). When the latter IZO NCs (with In content $\sim 3 \%$ ) were spin-coated, the prepared film had a poorer conductivity compared to that from IZO NCs synthesized by using $\mathrm{Zn}(\mathrm{St})_{2}$ precursor. 

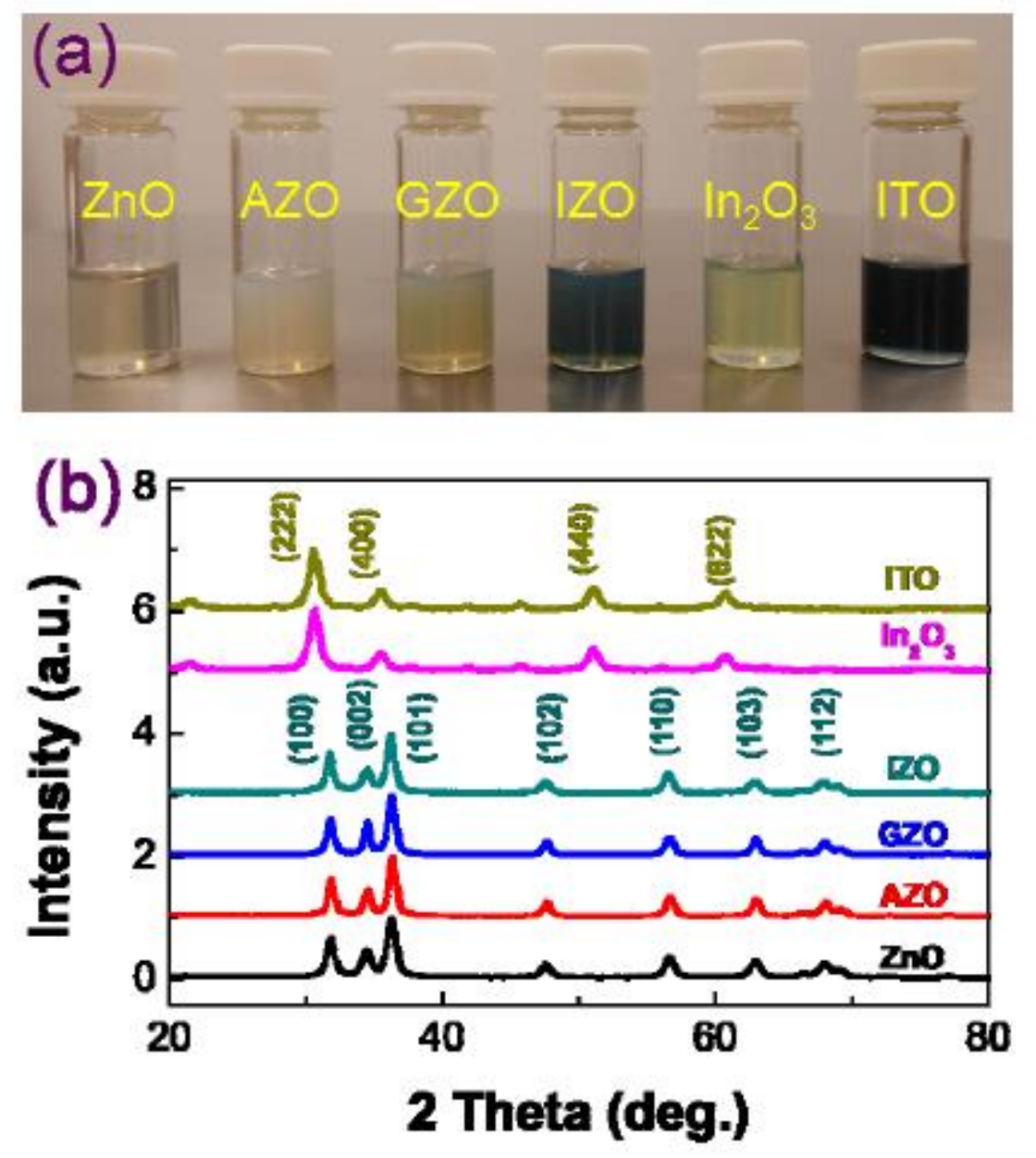

Figure 3 | TCO NCs inks. (a) Photograph of a series of TCO NC inks dispersed in toluene. (b) XRD of as-prepared TCO NCs. The original precursor content for AZO, GZO, IZO and ITO NCs was 1, 3, 5 and 5 at.\%, respectively.

The TCO NCs synthesized under optimal conditions were easily separated via centrifugation and then re-dispersed in various organic liquids, making inks ready for further film preparation through various wet-chemistry deposition techniques. Figure 3a shows the TCO NC inks dispersed in toluene which were proved to be stable for one year. When $\mathrm{Al}, \mathrm{Ga}$, In and $\mathrm{Sn}$ were incorporated into $\mathrm{ZnO}$ or $\operatorname{In}_{2} \mathrm{O}_{3}$ $\mathrm{NCs}$, the ink color changed from transparent (for $\mathrm{ZnO}$ ) to milky (AZO), green-yellow (GZO) and navy blue (IZO), or from yellow (for $\operatorname{In}_{2} \mathrm{O}_{3}$ ) to blue (ITO). The change is attributed to the dopants providing free electrons to the conduction band of TCO, the latter electrons being responsible for the surface plasmon resonance in the near-infrared region ${ }^{38,39}$. The XRD patterns presented in Fig. $3 \mathrm{~b}$ indicate 
that the doped $\mathrm{ZnO}$ and $\mathrm{In}_{2} \mathrm{O}_{3} \mathrm{NCs}$ preserve either the hexagonal $\mathrm{ZnO}$ wurtzite or the basic cubic $\operatorname{In}_{2} \mathrm{O}_{3}$ bixbyite structures, respectively. No additional phases, but those of $\mathrm{ZnO}$ or $\operatorname{In}_{2} \mathrm{O}_{3}$, were found irrespective of dopants or concentrations thereof used in this work.

Scaling up TCO NC preparation. A large-scale preparation experiment was carried out using a 5\% $\mathrm{Sn}(\mathrm{acac})_{2} \mathrm{Cl}_{2}$ and $95 \% \mathrm{In}(\mathrm{acac})_{3}$ mixture of precursors (Supplementary Note 1). This resulted in $2.1 \mathrm{~g}$ of ITO NCs as dry powder, which is ten times more than in the original experiments (Fig. 4a and Supplementary Fig. S7). Theoretically, if the reaction yield were 100\%, the mass of pure ITO would be just $1.45 \mathrm{~g}$, and thus the observed mass increment was due to surface OAm and OA molecules, as discussed above and as confirmed by IR spectra (Supplementary Fig. S8). As a result, the assynthesized NC powders obtained by drying in a vacuum box at room temperature can be easily redispersed in various organic solvents such as, e.g., toluene and hexane (Fig. 4b). Figure 4c gives a TEM image of the ITO NCs prepared at larger scale, where the average particle diameter is seen to be of $9 \pm 2.4 \mathrm{~nm}$. The morphology of the NCs in Fig. 4c is same as that in Figs. 1k,1 and only their size distribution is slightly larger (Supplementary Fig. S9). Thus, the proposed one-pot preparative method is indeed generic (as NC inks of various TCO materials can be universally prepared via the same strategy), simple and amenable to large-scale production, yet being not sensitive to the complexity of mixing characteristic of the hot-injection method. The obtained NC inks can be easily assembled into films via routine solution-based processes like, e.g., spin coating. When re-dispersed in proper solvents, such as n-tetradecane or n-tetradecane/hexane, the NC inks may be potentially used for the preparation of OEDs via ink-jet printing or roll-to-roll methods. In this case, the ink consumption rate can be significantly reduced, which is important when it comes to the preparation of OEDs containing the rare element In. Using the ITO NC ink prepared at larger scale and then re-dispersed, we prepared a 
2-layer ITO film with a thickness of $300 \mathrm{~nm}$. Its transparency and conductivity were very close to those of the film prepared from ITO NCs prepared at lower scale (Supplementary Fig. S10).

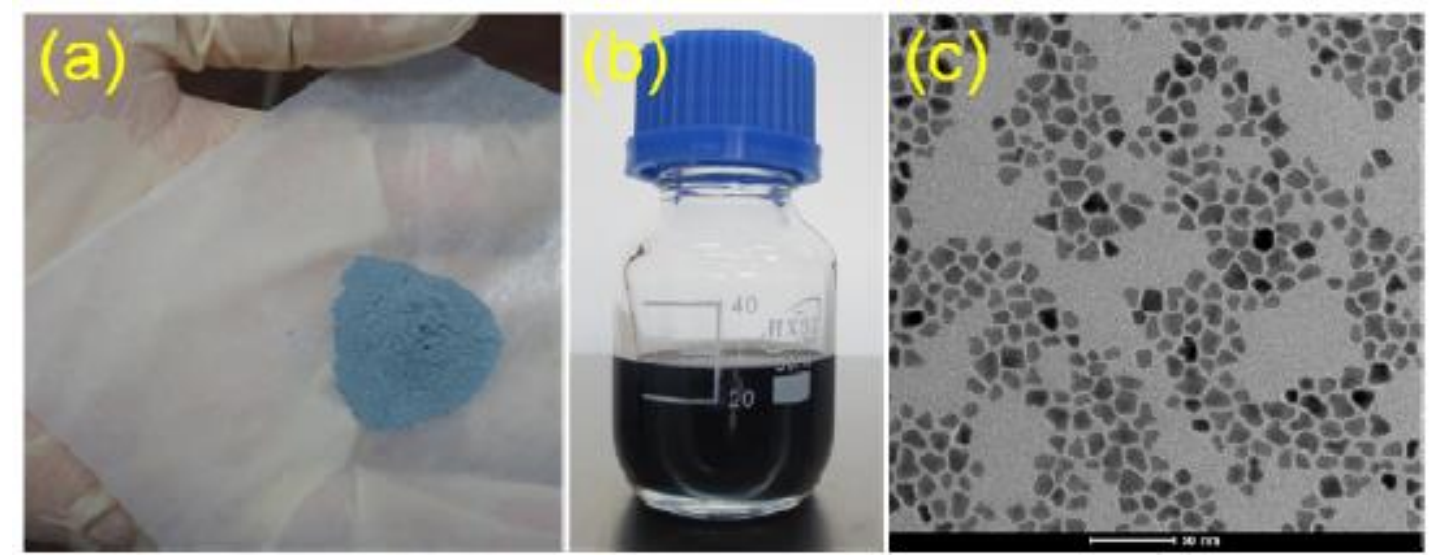

Figure 4 | Large-scale prepared ITO NCs. (a) Powder of ITO NCs prepared by one-pot method at larger scale ( $2.1 \mathrm{~g}$ ). (b) ITO NC ink prepared by re-dispersing dry powder in toluene. (c) TEM of ITO NCs prepared at larger scale.

TCO films prepared from inks. The NC inks shown in Fig. 3a can be used to deposit TCO NC thin films of TCO materials. For example, they could be easily spin-coated on glass substrates. Figure 5a shows cross-section of a 145-nm thick film prepared upon spin-coating one layer of an IZO NC ink in toluene. The film was treated by UV light for $60 \mathrm{~min}$, after which a second layer was spin-coated. The two-layer IZO film is seen in Fig. $5 \mathrm{~b}$ to be about $300 \mathrm{~nm}$ in thickness. Upon repeating the procedures one more time, a three-layer film of $460 \mathrm{~nm}$ in thickness was obtained (Fig. 5c). The large-scale crosssectional SEM image of the three-layer film (Fig. 5d) shows its dense, uniform and crack-free morphology and very smooth surface, which is also confirmed by a surface image in Fig. 5e. The rootmean-square surface roughness of the film was measured by atomic force microscopy (AFM) over the area of $5 \times 5 \mu \mathrm{m}^{2}$ to be $1.6 \mathrm{~nm}$ (Fig. 5f). In this study, various TCO films were successfully deposited by spin-coating inks of AZO, GZO, IZO, $\mathrm{In}_{2} \mathrm{O}_{3}$, and ITO NCs (see surface images in Supplementary Fig. S11). 

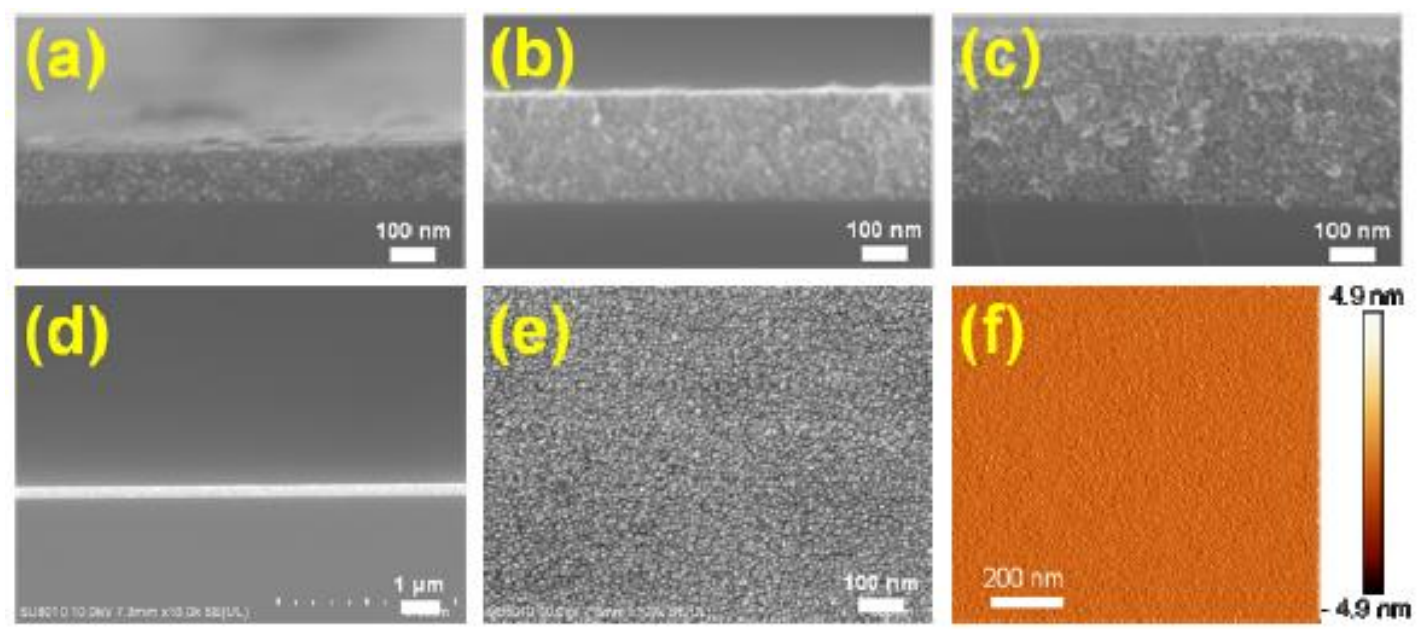

Figure 5 | Cross-section and surface images of IZO films. (a-c) SEM cross-section images of IZO NC films spincoated as one layer (a), two layers (b) and three layers (c) on glass substrates. (d) Large-scale cross-sectional view of the three-layer IZO film in (c). (e) SEM surface image and (f) AFM surface image of the three-layer IZO film in (c). Root-mean-square surface roughness based on (f) equals $1.6 \mathrm{~nm}$.

Optical and electrical properties of TCO films. The doping level of films with the lowest electrical resistance was found to be $1,3,5$ and $5 \%$ for AZO, GZO, IZO and ITO NCs, respectively. These values were obtained by plotting the sheet resistance versus doping level for films spin-coated using the above $\mathrm{NC}$ inks and then annealed in $\mathrm{Ar}$ with $5 \%$ of $\mathrm{H}_{2}$ at $350{ }^{\circ} \mathrm{C}$ for $3 \mathrm{~h}$ (Supplementary Fig. S12). The content of dopant elements incorporated into $\mathrm{ZnO}$ or $\mathrm{In}_{2} \mathrm{O}_{3} \mathrm{NCs}$ was found by energy-dispersive X-ray spectroscopy, XPS and ICP-AES to be slightly higher than in the precursor mixtures used to prepare the NCs (Supplementary Table S1). For instance, in $\mathrm{ZnO}$ doped with $3 \%$ of Ga, depending on analytic technique and considering only metal atoms, from 4.1 to 4.8 at. $\%$ of Ga was detected. Though the measured values still need further explanation, the results of chemical analyses clearly show that the proposed approach is capable of efficiently controlling the doping level of produced NCs.

In order to prepare films with optimum performance, we compared the sheet resistance of IZO films with different numbers of spin-coated layers (Supplementary Fig. S13). Similar to the other multilayered films, the samples were annealed in $\mathrm{Ar} / \mathrm{H}_{2}$ upon spinning each layer. The sheet resistance of the one-layer IZO film, with the highest transparency (Supplementary Fig. S14), was found to be 
larger, while the two-layer (300 nm-thick) film demonstrated the best combination of lower sheet resistance and high transparency (Supplementary Fig. S13). All the TCO films prepared in this study were highly transparent in the visible spectral range. Figure 6a presents the UV-Vis spectra of several two-layer films (300 nm-thick) and compares them with an uncoated glass substrate. All the films exhibit transparency which is slightly lower than that of the glass substrate. Nevertheless, their transmittance is seen in Fig.6a to be always over $80 \%$. In parallel, they also demonstrated high conductive properties. The optimally treated 300-nm-thick IZO and ITO films are seen in Fig.6b to demonstrate sheet resistance values as low as 1700 and $960 \Omega /$ sq, respectively. These values are still quite far from those anticipated for commercial applications. However, after additional UV treatment for $1 \mathrm{~h}$, they could be improved, reaching the values of 354 and $112 \Omega /$ sq, respectively. These values (<400 $\Omega / \mathrm{sq}$ ), along with high transparency of the IZO and ITO NC films, already meet the requirements for electrodes used in resistive touch panels ${ }^{6,40}$, or even in displays ${ }^{41,42}$ and lowtemperature coatings ${ }^{22,43}$. Though the other films prepared from TCO NC inks (AZO, GZO, as well as undoped $\mathrm{ZnO}$ and $\mathrm{In}_{2} \mathrm{O}_{3}$ ) demonstrated larger resistance values (Fig. 6b), their properties are very likely to be improved with further optimization, as other ink solvents, spin-coating parameters and post-deposition treatments can be applied.

Though the improvement in the electrical conductivity of TCO films has been observed and reported before ${ }^{22}$, its mechanism is not well-understood to date. In general, the conductivity of TCOs is known to originate from their intrinsic oxygen vacancies and/or extrinsic defects caused by doping donor. For the same sample, the donor concentration remains constant, and it is therefore the intrinsic oxygen vacancies that should be the main factor. The enhanced film conductivity observed after UV treatment can probably be attributed to an increased density of oxygen vacancies or to enhanced dopant 
activation. Figure $6 \mathrm{c}$ compares both the transparency and sheet resistance of various TCO films prepared by others via wet-chemistry routes with those prepared in the present report. It is well seen in Fig. 6c that the films prepared by spin-coating TCO NC inks in this study compare favorably with (and are often superior to) those previously reported in the literature. Therefore, the preparative technique described in this study, being amenable to scale up, truly shows potential in preparing highquality TCO NC inks comparable with their best counterparts reported in the literature, and thus is very attractive for numerous applications in solution-processed OEDs.

The outstanding optical and electrical properties demonstrated by the proper annealed ITO NC films can be potentially applied in solution-processed light-emitting diodes (LEDs). Although LEDs based on small phosphorescent organic molecules exhibit high electroluminescent efficiency, the vacuum thermal evaporation technique used for their preparation is not compatible with ink-jet printing and roll-to-roll mass production. Therefore, we fabricated fluorescent polymer light-emitting diodes (PLEDs) based on ITO NC film electrodes. This device structure is compatible with solution-processed techniques, such as roll-to-roll and ink-jet printing, and has potential applications in low-cost OEDs. The prepared device (schematically presented in Fig. S15b ) consisted of several layers: glass; ITO NC film; PEDOT:PSS; PF-3,7-FSO10; PFN and LiF/A1. Except for the LiF and Al cathode layer, which was deposited by thermal evaporation in vacuum, all the other layers were sequentially deposited onto a glass substrate using spin-coating. Figure 6d, where normalized electroluminescence spectra of the as-prepared PLED device and its commercially available counterpart are compared, exhibits that the spectrum of the ITO film-based device prepared from an ITO NC ink agrees well with that of its commercial counterpart that uses a sputter-deposited ITO electrode. The inset in Fig.6d shows that the device with a spin-coated ITO NC film as anode, when biased at $6 \mathrm{~V}$, emits a uniform 
blue light all over the pixel area. This provides additional confirmation that the ITO film was prepared smooth and crack-free over all its area. Although the electrical properties and efficiency (Supplementary Figs. S15 and S16) of PLED devices based on our ITO NC films are slightly below those for commercial ITO electrodes, such TCO NC-based films, especially upon further optimization of their properties, are believed to have a potential in various solution-processed OEDs due to their low costs and easy large-scale preparation.
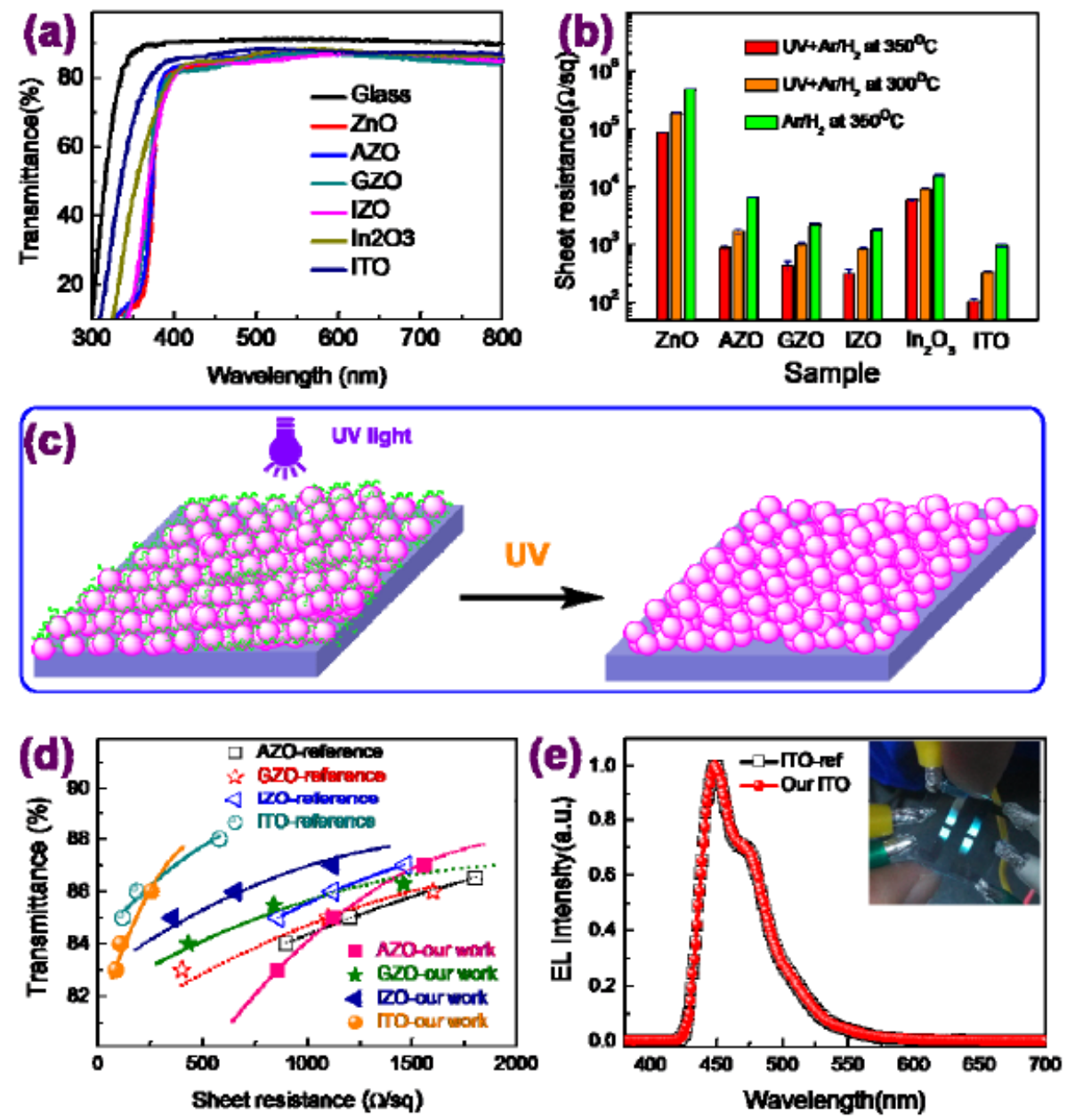

Figure 6 | Photoelectric properties of TCO NC films. (a) Transmittance of glass substrate and TCO NC films on glass substrate in the wavelength range from 300 to $800 \mathrm{~nm}$. (b) Sheet resistance of TCO NC films on glass substrates. The films with thickness of $300 \mathrm{~nm}$ were prepared by spin-coating, then treated by $\mathrm{UV}$, and then in $\mathrm{Ar}+5 \% \mathrm{H}_{2}$ at 350 ${ }^{\circ} \mathrm{C}$ for $3 \mathrm{~h}$. (c) Transmittance vs. sheet resistance of TCO NC films prepared in this study (filled symbols) and of those prepared by others: $\mathrm{AZO}^{22}, \mathrm{GZO}^{22}, \mathrm{IZO}^{44,45}$ and $\mathrm{ITO}^{18}$. (d) Normalized electroluminescence spectra of PLEDs with ITO NC film electrode prepared in this study and with commercial magnetron-sputtered ITO film. Inset: photograph of the device emitting blue light. 


\section{Discussion (Conclusion)}

In summary, a generic single-step method was developed to prepare NC inks of diverse metal-oxide materials, such as AZO, GZO, IZO, and ITO. An inexpensive long-chain alkyl-alcohol (1-dodecanol) is used in the method instead of more widely used 1,2-hexadecanediol. The prepared metal-oxide NCs were monodisperse and could be separated and then re-dispersed in various organic solvents to be used further as inks for thin-film fabrication. The thickness of such transparent oxide films was effectively controlled via the number of layers, while their conductivity could be enhanced by UV treatment. Twolayer IZO and ITO films with thickness of $300 \mathrm{~nm}$ annealed at $350{ }^{\circ} \mathrm{C}$ for $3 \mathrm{~h}$ showed improved sheet resistivity values as low as 354 and $112 \Omega /$ sq and transmittance as high as 84 and $87 \%$, respectively. The optimal performance of the films prepared from NC inks was comparable with that of state-ofthe-art films deposited from hot-injection-prepared inks. The proposed one-pot heating method is shown to be much more amenable to large-scale production, thus making the as-prepared NC inks very attractive for applications in solution-processed and flexible OEDs.

\section{- Methods}

Chemical reagents. Zinc stearate $\left(\mathrm{Zn}(\mathrm{St})_{2}\right.$, from Aladdin-reagent), aluminium triacetylacetone $\left(\mathrm{Al}(\mathrm{acac})_{3}, 98 \%\right.$ pure, from Aladdin-reagent), gallium acetylacetonate ( $\mathrm{Ga}(\mathrm{acac})_{3}, 99.99 \%$ pure, from Aladdin-reagent), indium (III) acetylacetonate $\left(\operatorname{In}(\mathrm{acac})_{3}, 99 \%\right.$ pure, from J\&K), tin (IV) bis(acetylacetonate) dichloride ( $\mathrm{Sn}(\mathrm{acac})_{2} \mathrm{Cl}_{2}, 98 \%$ pure, from Sigma-Aldrich), oleylamine (OAm, 80 90\% pure, from Aladdin-reagent), oleic acid (OA, from Aladdin-reagent), 1-octadecene (ODE, >80\% pure, from Aladdin-reagent), and 1-decanol (DDL, >99\% pure, from Aladdin-reagent) were used as received.

Synthesis of TCO NCs. We carried out a set of reactions starting with a certain concentration of each metal-containing precursor. In those reactions, the cation precursor quantity, which was the total amount of the matrix-metal precursor and the dopant-metal precursor, was kept at $1 \mathrm{mmol}$. Considering 
the transparency and conductivity of resulting films, the optimal molar ratios of metals for AZO, GZO, IZO, and ITO NCs were found to be 1:97 ( $\left.\mathrm{Al}(\mathrm{acac})_{3}: \mathrm{Zn}(\mathrm{St})_{2}\right), 3: 95\left(\mathrm{Ga}(\mathrm{acac})_{3}: \mathrm{Zn}(\mathrm{St})_{2}\right), 5: 95$ $\left(\operatorname{In}(\operatorname{acac})_{3}: \mathrm{Zn}(\mathrm{St})_{2}\right)$, and 5:95 $\left(\mathrm{Sn}(\operatorname{acac})_{2} \mathrm{Cl}_{2}: \operatorname{In}(\operatorname{acac})_{3}\right)$, respectively.

As an example, the synthesis protocol for the IZO NCs was as follows: $\mathrm{Zn}(\mathrm{St})_{2}(600 \mathrm{mg}, 0.95 \mathrm{mmol})$, $\operatorname{In}(\mathrm{acac})_{3}(20 \mathrm{mg}, 0.05 \mathrm{mmol}), 17 \mathrm{~mL}$ of ODE, $3 \mathrm{~mL}$ of OAm, $1 \mathrm{~mL}$ of OA and $1 \mathrm{~mL}$ of DDL were loaded in a $100 \mathrm{~mL}$ four-neck flask, degassed at $140{ }^{\circ} \mathrm{C}$ for $30 \mathrm{~min}$ and heated to $270{ }^{\circ} \mathrm{C}$ for $10 \mathrm{~min}$ under Ar flow. The mixture was kept at $270{ }^{\circ} \mathrm{C}$ for $30 \mathrm{~min}$, after which the mixture was cooled to room temperature by a water bath. The resultant NCs were precipitated by a mixture of $5 \mathrm{~mL}$ of hexane and $25 \mathrm{~mL}$ of ethanol and separated via centrifugation. The separated NCs were re-dispersed in $20 \mathrm{~mL}$ of hexane in the presence of OAm $(0.2 \mathrm{~mL})$ and $\mathrm{OA}(0.2 \mathrm{~mL}) .30 \mathrm{~mL}$ of ethanol was added to flocculate the NCs, which were then separated by centrifugation. The process was repeated once more, and the final product was dispersed in $\sim 2.5 \mathrm{~mL}$ of toluene for further use. To optimize the optical and electrical properties of doped films, TCO NCs with different dopant fractions were prepared. The dopant concentration in TCO NCs resulting in films with optimal performance was found to be $1,3,5$ and 5 at.\% for AZO, GZO, IZO and ITO, respectively.

Preparation of TCO NC films. Glass substrates $\left(20 \times 20 \mathrm{~mm}^{2}\right)$ were first cleaned twice in acetone via sonication for $15 \mathrm{~min}$. Then the substrates were annealed at $200{ }^{\circ} \mathrm{C}$ for $30 \mathrm{~min}$ and treated with UV light for $15 \mathrm{~min}$. A total of $0.12 \mathrm{~mL}$ of TCO NC dispersion in toluene was spin-coated onto the substrate at 3,000 rpm for $50 \mathrm{~s}$. To increase the film thickness, the films were prepared in 2-3 steps, and each layer was UV-treated after deposition to enhance its surface smoothness and electrical properties. In order to elucidate the effect of UV treatment, some samples treated by UV light were annealed in a $\mathrm{H}_{2} / \mathrm{Ar}(5: 95 \mathrm{v} / \mathrm{v})$ mixture at a certain temperature for $3 \mathrm{~h}$ in a tube furnace. Samples untreated by UV irradiation were annealed at $250{ }^{\circ} \mathrm{C}$ prior to depositing a next layer.

Fabrication of PLEDs. A poly(dibenzothiophene-S,S-dioxide-co- 9,9-dioctyl-2,7-fluorene) copolymer ( PF-3,7-FSO10, JiLin OLED Material Tech Co., Ltd., $\mathrm{M}_{\mathrm{w}}=101,000$ g/mol, chemical structure shown in Supplementary Fig. S17 ) was used for the PLED emission layer ${ }^{46}$. A poly [(9,9bis 3'-(N,N-dimethylamino)propyl)-2,7-fluorene)-alt-2,7-(9,9-dioctylfluorene)] (PFN, JiLin OLED Material Tech Co., Ltd., chemical structure shown in Supplementary Fig. S17) was used for the PLED electron transport layer ${ }^{47}$. PEDOT:PSS (Baytron P VPAI 4083, H. C. Starck) was used for the holetransporting layer in the PLEDs. For regular devices, a 40-nm-thick PEDOT:PSS anode buffer layer 
was spin-coated onto the ITO substrate, then dried at $80{ }^{\circ} \mathrm{C}$ for $30 \mathrm{~min}$. The emission layer was prepared by spin-coating PF-3,7-FSO10 chlorobenzene solution (concentration: $7 \mathrm{mg} / \mathrm{mL}$ ) at 3,000 rpm for 1 min. The PFN was dissolved in methanol in the presence of a small amount of acetic acid (2 $\mu \mathrm{L} / \mathrm{mL}$ ) and its solution (concentration: $3 \mathrm{mg} / \mathrm{mL}$ ) was spin-coated on top of the emission layer. A 1nm-thick LiF layer and a 100-nm-thick Al layer were subsequently evaporated through a shadow mask to define the active area of the devices $\left(2 \times 1.5 \mathrm{~mm}^{2}\right)$.

Characterization Techniques. One drop of the diluted as-synthesized TCO NC dispersion was placed onto a carbon-coated $\mathrm{Cu}$ grid, and toluene was allowed to evaporate at room temperature. TEM and HRTEM images and energy-dispersive X-ray spectroscopy (EDX) were taken on a Tecnai G2 F30 STWIN TEM instrument operated at an acceleration voltage of $200 \mathrm{kV}$ and equipped with an EDX/STEM HAADF detector. XRD samples were prepared by dropping concentrated toluene dispersions of purified NCs onto glass substrates. XRD patterns were acquired using a Bruker D8 Advance X-ray diffractometer operating with $\mathrm{Cu} \mathrm{K \alpha}$ radiation $(\lambda=1.5406 \AA$ ). Surface and crosssectional SEM images of the TCO NC films were obtained on a SU 8010 FE-SEM instrument. To evaluate film roughness via AFM, TCO NCs were spin-coated onto Si substrates and then analyzed by a Veeco D3100 AFM tool. Composition of TCO NCs was analyzed by ICP-AES with a Varian 720/730 Series spectrometer. The samples were dissolved in concentrated $\mathrm{HCl} / \mathrm{HNO}_{3}(3: 1 \mathrm{v} / \mathrm{v})$. Sheet resistance of the TCO NC assemblies was obtained by means of four-point probe measurements and the van der Pauw method over 20x20 mm² areas on glass substrates at room temperature, and the final values were averaged over a minimum of five measurements for each sample. The transmittance of TCO NC films on glass substrates was measured by a Shimadzu UV-3600 UV/VIS/NIR spectrophotometer (in the $250-800 \mathrm{~nm}$ spectral range). FTIR measurements of samples deposited onto Si substrates were performed using a Shimadzu IR Prestige-21 instrument, with a resolution of $4 \mathrm{~cm}^{-1}$. XPS spectra were recorded on a Thermo ESCALAB-250 spectrometer using a monochromatic Al KR radiation source $(1486.6 \mathrm{eV})$. XPS samples were prepared by dropping concentrated toluene dispersions of purified NCs onto Si wafers. The EL spectra and luminance (L)-current density (J)luminance efficiency (LE) characteristics were collected by using a Keithley 2400 source and a PR705 SpectraScan spectrophotometer (Photo Research) in air and at room temperature. 


\section{Acknowledgments}

This work was supported by the National 973 project from National Basic Research Program of China (2014CB931700), the National Natural Science Foundation of China (61222403 and 11274173), the Doctoral Program Foundation from the Ministry of Education of China (20123218110030), the Fundamental Research Funds for the Central Universities (30920130111017 and NE2012004) and the Opened Fund of the State Key Laboratory on Integrated Optoelectronics (IOSKL2012KF06).

\section{Author contributions}

J.Z.S. and H.B.Z. designed the experiments, interpreted the data and co-wrote the paper. J.Z.S. and Y.L.L. performed synthesis of nanocrystals, their assembling into thin films and characterization. J.Z.S. designed and fabricated the PLED devices. S.A.K. revised the manuscript. All authors discussed and commented on the manuscript.

\section{Additional information}

Supplementary Information accompanies this paper at http://www.nature.com/naturecommunications

Reprints and permission information is available online at http://npg.nature.com/reprintsandpermissions/

\section{- References}

1. He, Z.C., Zhong, C.M., Su, S.J., Xu, M., Wu, H.B., Cao, Y. Enhanced power-conversion efficiency in polymer solar cells using an inverted device structure. Nat. Photonics 6, 591-595 (2012).

2. Choi, H., et al. Versatile surface plasmon resonance of carbon-dot-supported silver nanoparticles in polymer optoelectronic devices. Nat. Photonics 7, 732-738 (2013).

3. Minami, T. Transparent conducting oxide semiconductors for transparent electrodes. Semicond. Sci. Tech. 20, S35S44 (2005).

4. Ginley, D.S., Bright, C. Transparent conducting oxides. MRS Bull. 25, 15-18 (2000).

5. Luo, L., Bozyigit, D., Wood, V., Niederberger, M. High-Quality Transparent Electrodes Spin-Cast from Preformed Antimony-Doped Tin Oxide Nanocrystals for Thin Film Optoelectronics. Chem. Mater. 25, 4901-4907 (2013).

6. Wu, H., et al. A transparent electrode based on a metal nanotrough network. Nat. Nanotechnol. 8, 421-425 (2013).

7. $\mathrm{Xu}, \mathrm{F}$, Zhu, Y. Highly Conductive and Stretchable Silver Nanowire Conductors. Adv. Mater. 24, $5117-5122$ (2012).

8. Guo, H.Z., et al. Copper Nanowires as Fully Transparent Conductive Electrodes. Sci. Rep. 3, 2323 (2013).

9. Fortunato, E., Ginley, D., Hosono, H., Paine, D.C. Transparent conducting oxides for photovoltaics. MRS Bull. 32, 242-247 (2007).

10. Kim, H., Horwitz, J.S., Qadri, S.B., Chrisey, D.B. Epitaxial growth of Al-doped ZnO thin films grown by pulsed laser deposition. Thin Solid Films 420, 107-111 (2002).

11. Asahi, R., Morikawa, T., Ohwaki, T., Aoki, K., Taga, Y. Visible-light photocatalysis in nitrogen-doped titanium oxides. Science 293, 269-271 (2001). 
12. Shen, G.Z., Xu, J., Wang, X.F., Huang, H.T., Chen, D. Growth of Directly Transferable $\operatorname{In}_{2} \mathrm{O}_{3}$ Nanowire Mats for Transparent Thin-film Transistor Applications. Adv. Mater. 23, 771-775 (2011).

13. Cui, J., et al. Indium tin oxide alternatives - High work function transparent conducting oxides as anodes for organic light-emitting diodes. Adv. Mater. 13, 1476-1480 (2001).

14. Musil, J., Vlček, J. A perspective of magnetron sputtering in surface engineering. Surf. Coat. Technol. 112, 162-169 (1999).

15. Hong, H., Jung, H., Hong, S.-J. Recycling of the indium scrap from ITO sputtering waste. Res. Chem. Intermed. 36, 761-766 (2010).

16. Jeong, J.-A., Lee, J., Kim, H., Kim, H.-K., Na, S.-I. Ink-jet printed transparent electrode using nano-size indium tin oxide particles for organic photovoltaics. Sol. Energ. Mat. Sol. C. 94, 1840-1844 (2010).

17. Buhler, G., Tholmann, D., Feldmann, C. One-pot synthesis of highly conductive indium tin oxide nanocrystals. $A d v$. Mater. 19, 2224-2227 (2007).

18. Lee, J., Lee, S., Li, G., Petruska, M.A., Paine, D.C., Sun, S. A Facile Solution-Phase Approach to Transparent and Conducting ITO Nanocrystal Assemblies. J. Am. Chem. Soc. 134, 13410-13414 (2012).

19. Chen, Y.F., Kim, M., Lian, G., Johnson, M.B., Peng, X.G. Side reactions in controlling the quality, yield, and stability of high quality colloidal nanocrystals. J. Am. Chem. Soc. 127, 13331-13337 (2005).

20. Liu, X., Swihart, M.T. A general single-pot heating method for morphology, size and luminescence-controllable synthesis of colloidal ZnO nanocrystals. Nanoscale 5, 8029-8036 (2013).

21. Narayanaswamy, A., Xu, H.F., Pradhan, N., Kim, M., Peng, X.G. Formation of nearly monodisperse $\mathrm{In}_{2} \mathrm{O}_{3}$ nanodots and oriented-attached nanoflowers: Hydrolysis and alcoholysis vs pyrolysis. J. Am. Chem. Soc. 128, 10310-10319 (2006).

22. Della Gaspera, E., et al. Low-Temperature Processed Ga-Doped ZnO Coatings from Colloidal Inks. J. Am. Chem. Soc. 135, 3439-3448 (2013).

23. Hyeon, T. Chemical synthesis of magnetic nanoparticles. Chem. Commun., 927-934 (2003).

24. Peng, X.G., et al. Shape control of CdSe nanocrystals. Nature 404, 59-61 (2000).

25. Chang, J., et al. ZnO Nanocones with High-Index $\{10 \overline{1} 1\}$ Facets for Enhanced Energy Conversion Efficiency of DyeSensitized Solar Cells. J. Phys. Chem. C 117, 13836-13844 (2013).

26. Wang, T., Chirmanov, V., Chiu, W.H.M., Radovanovic, P.V. Generating Tunable White Light by Resonance Energy Transfer in Transparent Dye-Conjugated Metal Oxide Nanocrystals. J. Am. Chem. Soc. 135, 14520-14523 (2013).

27. Chen, T., Tang, K. $\gamma-\mathrm{Ga}_{2} \mathrm{O}_{3}$ quantum dots with visible blue-green light emquantum dots with visible blue-green light emission property. Appl. Phys. Lett. 90, 053104 (2007).

28. Wang, T., Farvid, S.S., Abulikemu, M., Radovanovic, P.V. Size-Tunable Phosphorescence in Colloidal Metastable $\gamma$ $\mathrm{Ga}_{2} \mathrm{O}_{3}$ Nanocrystals. J. Am. Chem. Soc. 132, 9250-9252 (2010).

29. Binet, L., Gourier, D. Orgin of the Blue Luminescence of $\quad \beta-\mathrm{Ga}_{2} \mathrm{O}_{3}$. J. Phys. Chem. Solids 59, 1241-1249 (1998).

30. Zhong, X., Feng, Y., Zhang, Y., Lieberwirth, I., Knoll, W. Nonhydrolytic Alcoholysis Route to MorphologyControlled ZnO Nanocrystals. Small 3, 1194-1199 (2007).

31. Joo, J., Kwon, S.G., Yu, J.H., Hyeon, T. Synthesis of ZnO Nanocrystals with Cone, Hexagonal Cone, and Rod Shapes via Non-Hydrolytic Ester Elimination Sol-Gel Reactions. Adv. Mater. 17, 1873-1877 (2005).

32. Narayanaswamy, A., Xu, H., Pradhan, N., Kim, M., Peng, X. Formation of Nearly Monodisperse $\operatorname{In}_{2} \mathrm{O}_{3} \mathrm{Nanodots}$ and Oriented-Attached Nanoflowers: Hydrolysis and Alcoholysis vs Pyrolysis. J. Am. Chem. Soc. 128, 10310-10319 (2006).

33. Tienes, B.M., Perkins, R.J., Shoemaker, R.K., Dukovic, G. Layered Phosphonates in Colloidal Synthesis of Anisotropic ZnO Nanocrystals. Chem. Mater. 25, 4321-4329 (2013). 
34. Yang, Y.F., et al. Dopant-Induced Shape Evolution of Colloidal Nanocrystals: The Case of Zinc Oxide. J. Am. Chem. Soc. 132, 13381-13394 (2010).

35. Norris, D.J., Efros, A.L., Erwin, S.C. Doped Nanocrystals. Science 319, 1776-1779 (2008).

36. Buonsanti, R., Milliron, D.J. Chemistry of Doped Colloidal Nanocrystals. Chem. Mater. 25, 1305-1317 (2013).

37. Erwin, S.C., Zu, L., Haftel, M.I., Efros, A.L., Kennedy, T.A., Norris, D.J. Doping semiconductor nanocrystals. Nature 436, 91-94 (2005).

38. Wang, T., Radovanovic, P.V. Free Electron Concentration in Colloidal Indium Tin Oxide Nanocrystals Determined by Their Size and Structure. J. Phys. Chem. C 115, 406-413 (2011).

39. Buonsanti, R., Llordes, A., Aloni, S., Helms, B.A., Milliron, D.J. Tunable Infrared Absorption and Visible Transparency of Colloidal Aluminum-Doped Zinc Oxide Nanocrystals. Nano Lett. 11, 4706-4710 (2011).

40. Hecht, D.S., Hu, L., Irvin, G. Emerging Transparent Electrodes Based on Thin Films of Carbon Nanotubes, Graphene, and Metallic Nanostructures. Adv. Mater. 23, 1482-1513 (2011).

41. Fahland, M., Karlsson, P., Charton, C. Low resisitivity transparent electrodes for displays on polymer substrates. Thin Solid Films 392, 334-337 (2001).

42. Lewis, J., Grego, S., Chalamala, B., Vick, E., Temple, D. Highly flexible transparent electrodes for organic lightemitting diode-based displays. Appl. Phys. Lett. 85, 3450-3452 (2004).

43. Leterrier, Y., et al. Mechanical integrity of transparent conductive oxide films for flexible polymer-based displays. Thin Solid Films 460, 156-166 (2004).

44. Chen, K.J., Hung, F.Y., Chang, S.J., Hu, Z.S. Microstructures, optical and electrical properties of In-doped ZnO thin films prepared by sol-gel method. Appl. Surf. Sci. 255, 6308-6312 (2009).

45. Hammarberg, E., Prodi-Schwab, A., Feldmann, C. Microwave-assisted polyol synthesis of aluminium- and indiumdoped $\mathrm{ZnO}$ nanocrystals. J. Colloid Interf. Sci. 334, 29-36 (2009).

46. Li, Y., Wu, H., Zou, J., Ying, L., Yang, W., Cao, Y. Enhancement of spectral stability and efficiency on blue lightemitters via introducing dibenzothiophene-S,S-dioxide isomers into polyfluorene backbone. Org. Electron. 10, $901-$ 909 (2009).

47. Zeng, W., et al. Polymer light-emitting diodes with cathodes printed from conducting Ag paste. Adv. Mater. 19, 810814 (2007). 